\title{
Nonlinear Power Amplifier Effects on a Full Duplex Spatial Modulation System
}

\author{
Yanni Zhou \\ Univ Lyon, INSA Lyon, Inria, CITI \\ Villeurbanne, France \\ yanni.zhou@insa-lyon.fr \\ Guillaume Villemaud \\ Univ Lyon, INSA Lyon, Inria, CITI \\ Villeurbanne, France \\ guillaume.villemaud@insa-lyon.fr
}

\author{
Florin Hutu \\ Univ Lyon, INSA Lyon, Inria, CITI \\ Villeurbanne, France \\ florin-doru.hutu@insa-lyon.fr \\ Taneli Riihonen \\ Electrical Engineering, Tampere University \\ Tampere, Finland \\ taneli.riihonen@tuni.fi
}

\begin{abstract}
In this paper, we consider a full duplex (FD) system using spatial modulation (SM) technique for simultaneous transmission. Despite the recent studies on the combination of these two techniques, nonlinear effects of devices have been ignored. This paper demonstrates, for the first time, the nonlinear power amplifier (PA) effects on a full duplex spatial modulation (FDSM) system. A digital canceller is adopted to deal with the strong selfinterference (SI) in presence of PA nonlinearities. The results are verifying that the considered canceller can suppress SI close to the thermal noise level and has high reliability to ensure the performance of the system.
\end{abstract}

Index Terms-Full duplex, spatial modulation, nonlinear selfinterference cancellation, power amplifier, bit error rate.

\section{INTRODUCTION}

Nowadays, with the development of the advanced generation technology of mobile communication, the number of mobile terminals and devices has shown an exponential growth trend. It has brought considerable pressure to wireless communication networks. Currently, most mobile networks use half-duplex (HD) mode, which limits networks performance because it causes a waste of wireless spectrum resources. Hence, full duplex (FD) has been proposed [1] [2] to improve the spectrum efficiency. The FD technology can theoretically double the channel capacity of the communication system. However, the self-interference (SI) of FD system has always been the core issue.

To overcome the issue that the signal emitted by the node itself covers the received signal of interest, passive suppression and active cancellation have been analyzed. Passive suppression mainly refers to the spatial distance between the receiving and transmitting antennas, so that the SI signal can get an attenuation before being received. Analog cancellation introduces an analog reference signal from the transmitting chain, then sends it to the receiving antenna to balance the SI signal after adjusting the amplitude and phase delay. For digital cancellation, a digital reference signal is generated from the transmitting baseband and aims to cancel SI by recovering the residual SI signal after appropriate adjustments. Compared with analog cancellation, self-interference cancellation (SIC) in digital domain is more flexible and can make it more efficient [3].

Meanwhile, spatial modulation (SM) as a new MIMO technique has attracted wide attention in recent years [4]. The SM system has only one transmitting antenna activated for each time slot, therefore, only one radio frequency (RF) chain is needed. It combines modulation on spatial resources with conventional amplitude and phase modulation. In this way, it exploits the index of the transmitting antennas to convey additional information bits. Therefore, SM systems can avoid inter-channel interference, inter-antenna synchronization, and high complexity of detection in the traditional MIMO system. Moreover, due to the introduction of space resources, the system spectrum efficiency is guaranteed, so it has become a low-cost, low-complexity MIMO system.

In conclusion, compared with the conventional FD-MIMO system, full duplex spatial modulation (FDSM) system shows remarkable interest [5]. The combination of FD and SM will not only maintain the spectrum efficiency but also decrease the complexity of the SIC and of the entire receiver because of the single RF chain.

To guarantee the performance of the FDSM system, a high accuracy SIC should be ensured. Since the actual hardware is not as ideal as the theoretical assumptions, various distortions and noises will inevitably be introduced. It will cause a gap between the SI signal recovered by the traditional channel estimation and the actual SI signal, which restricts the performance of digital cancellation. In the literature, [6] shows the performance of a FD radio under nonideal RF components. Nonlinear canceller with complexity reduction scheme for FD radios is described in [7] [8]. Moreover, the impact of the receiver nonidealities including IQ imbalance and phase noise on a FDSM system is discussed in [9]. However, to the authors' knowledge, nonlinear effects on the FDSM system have been scarcely investigated from the perspective of power amplifier (PA).

In this paper, we analyze especially the nonlinear effects of PA and a digital canceller is employed to suppress the SI 


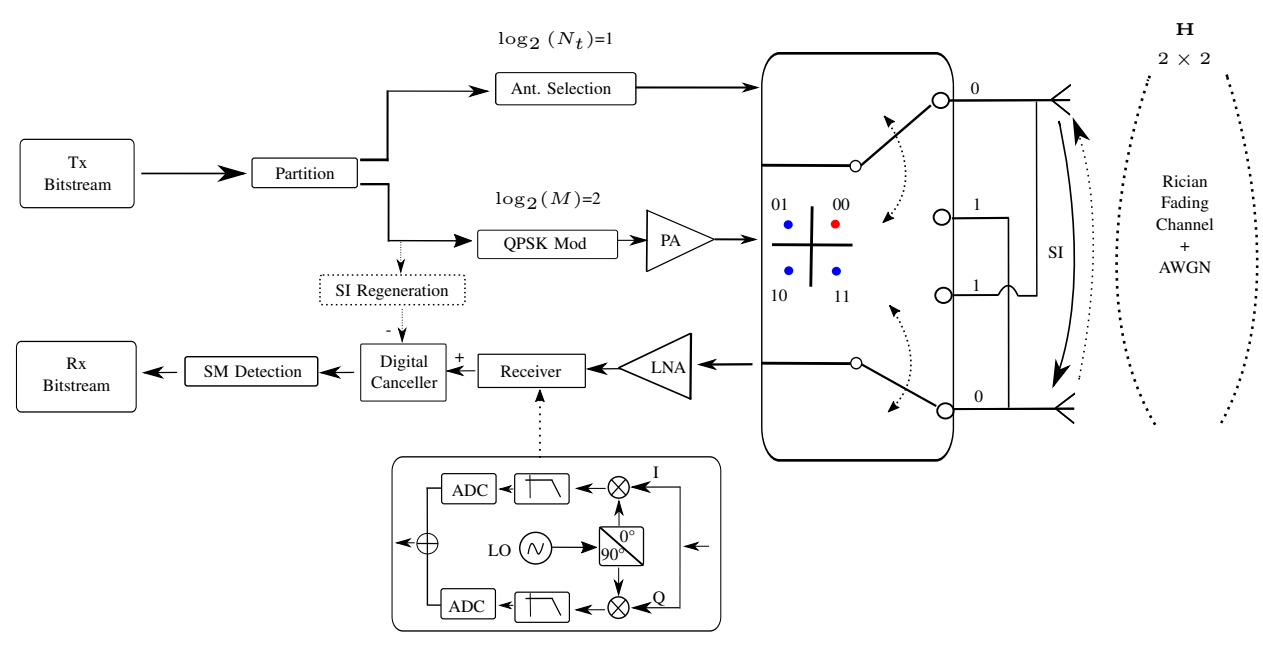

Fig. 1. $2 \times 2$ FDSM system model with a digital canceller: Node A

signal. The remainder of the paper is organized as follows: Section II presents the considered nonlinear FDSM system model, along with the considered digital canceller. The system level simulation results are discussed in Section III, after which conclusions and future directions are given in Section IV.

\section{System ARChiteCtURE AND Digital CANCELlER}

Due to the characteristics of FD communication system, both ends of the system have the same functions of simultaneous emitting and receiving. Consequently, in this article, we only show the structural characteristics of one end. The structure of Node A of a $2 \times 2$ FDSM system is illustrated in Fig. 1. For ease of understanding, we divide Node A into three parts for the detailed explanation.

\section{A. Transmitting part}

The input data is supposed to be a random binary sequence. Meanwhile, the input data will be saved as a file for the system performance analysis at the receiving part. The core technique of SM is the mapping mode of the binary sequences. The number of bits for antenna selection and symbol construction at each time slot is $\log _{2}\left(N_{t}\right)$ and $\log _{2}(M)$, respectively. $N_{t}$ corresponds to the number of the antennas at each node, $M$ represents the modulation order. The previous part is used to select one antenna for transmitting, through an antenna switch. The remainder is used to form the corresponding quadrature amplitude modulation (QAM) symbol. In our model, $N_{t}=2$ and $M=4$. More precisely, the antenna selected by the switch will transmit the QPSK symbols, and the other antenna will be used to receive the signal from the other node.

Before transmitted by the selected antenna, the modulated signal will be amplified by the PA which may impact the communication performance by its nonlinear behavior.

Nonlinear distortions produced by the transmitter's PA is one of the non-idealities of RF chain which become a significant issue in a FD transceiver when using cheaper and less
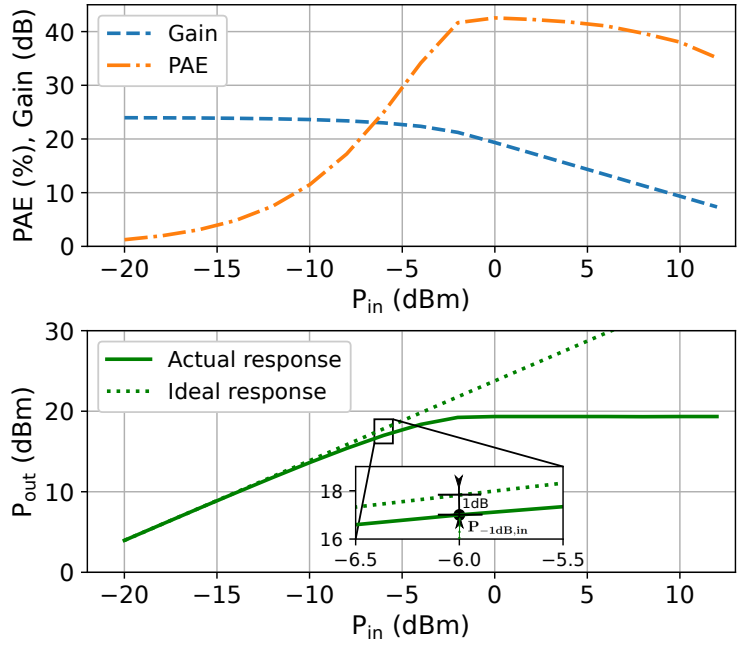

Fig. 2. CC2595 Typical Characteristics in simulation

linear power amplifier. In order to study this impact, in this paper we choose the low-cost PA of the type CC2595 from Texas Instruments [10] and apply its model in the FDSM's system level simulation. The CC2595 has a typical gain of 24 $\mathrm{dB}$, its operating frequency range is between $2400 \mathrm{MHz}$ and $2483.5 \mathrm{MHz}$ and the typical output power is $P_{\text {out }}=20.7 \mathrm{dBm}$. The simulation results of the CC2595 model are presented in Fig. 2. Here Gain represents the amplifier's gain in $\mathrm{dB} ; P_{\text {out }}$ denotes the output power and PAE represents the power-added efficiency which is a metric for rating the efficiency of a PA. As can be remarked from this figure, the $-1 \mathrm{~dB}$ compression point is obtained for input power levels of about $-6 \mathrm{dBm}$ and the corresponding output power level is $17 \mathrm{dBm}$, as presented in the CC2595's datasheet. In order to incorporate the PA's nonlinear effect, the performance of the canceller will be evaluated for PA's input power levels greater than $-6 \mathrm{dBm}$. 


\section{B. Channel environment}

In this paper, the main channel between two nodes is defined as a $2 \times 2$ sized Rician fading channel. Average white Gaussian noise (AWGN) $\eta$ is also taken into account. SI channel is supposed to be a single-path one delay channel where the strength of the direct path dominates the power of the SI channel. Besides, self-interference-to-noise ratio (INR) is introduced as the power ratio of the received SI signal to the receiver thermal noise, in order to evaluate the SI power. A large range ( $[-20 \mathrm{~dB}, 80 \mathrm{~dB}])$ INR is applied to represent the different levels of SI power for the receiving end. In a practical sense, we can understand the influence of the changes with INR as that of the changes with the distance between the two nodes on the performance of the system.

\section{Receiving part}

The signal received at node $\mathrm{A}\left(x_{n}^{a}\right)$ is the combination of two parts: one from node B and the other is SI. Precisely, the received signal $\left(s_{n}\right)$ from node $\mathrm{B}$ after passing the Rician fading channel and the SI $\left(s_{n}^{s i}\right)$ generated by the transmitting part from its own node (node A). The nonlinear signal distortion produced by PA has significant effects on SI signal. Here, the nonlinear signal model is supposed as a sample memoryless polynomial model. For higher accuracy, a model with memory such as parallel Hammerstein model can also be supposed as shown in [7] [11]. The signal $x_{n}^{a}$ received by the antenna can be given as follows.

$$
x_{n}^{a}=s_{n}+s_{n}^{s i}+\eta
$$

with the received SI signal $s_{n}^{s i}$ given as

$$
s_{n}^{s i}=h_{n} \cdot\left(s_{n}^{a}\right)_{P A}
$$

where $\eta$ corresponds to the AWGN noise, $h_{n}$ denotes the SI channel and $s_{n}^{a}$ is the input signal of PA at node A. $\left(s_{n}^{a}\right)_{P A}$ represents the baseband output signal of PA, given as

$$
\left(s_{n}^{a}\right)_{P A}=\sum_{k=0}^{K} p_{k} \cdot s_{n}^{a} \cdot\left|s_{n}^{a}\right|^{k}
$$

where $p_{k}$ is the complex-valued coefficient of the PA polynomial model and $K+1$ is the maximum order of the polynomial. In general, only odd-orders are considered because only oddorder distortion will appear around the received signal. Hence, the odd-order polynomial model can be presented as

$$
\begin{aligned}
\left(s_{n}^{a}\right)_{P A} & =\sum_{k=0}^{K} p_{k} \cdot s_{n}^{a} \cdot\left|s_{n}^{a}\right|^{2 k} \\
& =\sum_{k=0}^{K} p_{k} \cdot i_{2 k+1}\left(s_{n}^{a}\right)
\end{aligned}
$$

1) Receiver's radio front-end: The combined signal is transposed in baseband by an analog radio front-end. In practice, IQ imbalance which is the mismatch between the I path and $\mathrm{Q}$ path occurs. Moreover, the phase noise of the local oscillator is also a non-negligible factor that degrades the system performance. A previous work [9] has discussed the effects of these nonidealities on a FDSM system. IQ imbalance and phase noise are assumed ideal in this paper.

2) Digital canceller: As mentioned before, we assume the rest of the radio front-end is ideal to ensure the nonlinearities of the system as the single variable. Since the nonlinear distortion is mainly caused by the PA in the transmitting part [11], we do not have to take other minor nonlinear effects into account. A nonlinearity digital canceller is employed after the analog-to-digital conversion at the receiving part. The idea for this canceller is to regenerate an estimated SI signal. This estimator is based on the Least Square (LS) algorithm, the signal $\hat{x}_{n}$ after the cancellation is given as

$$
\hat{x}_{n}=x_{n}^{a^{*}}-\hat{s}_{n}^{s i^{*}}
$$

where $x_{n}^{a^{*}}$ is the digital signal after passing the radio front-end and $\hat{s}_{n}^{s i^{*}}$ is the estimated SI based on LS.

$$
\begin{aligned}
\hat{s}_{n}^{s i^{*}} & =\sum_{k=0}^{K} h_{n} \cdot \hat{p}_{k} \cdot i_{2 k+1}\left(s_{n}^{a}\right) \\
& =\sum_{k=0}^{K} \hat{w}_{k} \cdot i_{2 k+1}\left(s_{n}^{a}\right)
\end{aligned}
$$

The estimator aims to calculate the weights $\hat{w}_{k}$ which minimize the power of signal after SIC $\hat{x}_{n}$ in the digital domain, which guarantees the recovery of the signal of interest. $\hat{w}_{k}$ is assumed as the complex-valued coefficient of both the SI channel and the PA polynomial model and is given as

$$
\hat{\mathbf{w}}_{\mathbf{k}} \stackrel{\text { def }}{=}\left[\begin{array}{ll}
\hat{w}_{1} & \hat{w}_{3} \cdots \hat{w}_{2 K+1}
\end{array}\right]^{\mathbf{T}}
$$

The received signal matrix $\mathrm{x}^{\mathrm{a}^{*}}$ with $\mathrm{N}$ samples of estimation is presented as

$$
\mathbf{x}^{\mathbf{a}^{*}} \stackrel{\text { def }}{=}\left[\begin{array}{ll}
x_{n}^{a^{*}} & x_{n+1}^{a^{*}} \cdots x_{n+N-1}^{a^{*}}
\end{array}\right]^{\mathbf{T}}
$$

The input matrix $\mathbf{i}$ is given as

$$
\begin{aligned}
& \mathbf{i} \stackrel{\text { def }}{=}\left[\begin{array}{llll}
\mathbf{i}_{1} & \mathbf{i}_{3} & \cdots & \mathbf{i}_{2 K+1}
\end{array}\right] \\
& \mathbf{i}_{2 k+1}=\left[\begin{array}{l}
i_{2 k+1}\left(s_{n}^{a}\right) \\
i_{2 k+1}\left(s_{n+1}^{a}\right) \\
\vdots \\
i_{2 k+1}\left(s_{n+N-1}^{a}\right)
\end{array}\right]=\left[\begin{array}{l}
s_{n}^{a} \cdot\left|s_{n}^{a}\right|^{2 k} \\
s_{n+1}^{a} \cdot\left|s_{n+1}^{a}\right|^{2 k} \\
\vdots \\
s_{n+N-1}^{a} \cdot\left|s_{n+N-1}^{a}\right|^{2 k}
\end{array}\right]
\end{aligned}
$$

Then, we can obtain:

$$
\begin{aligned}
\hat{\mathbf{w}}_{\mathbf{k}}^{\mathbf{L S}} & =\underset{w_{k}}{\arg \min }\left\|\mathbf{x}^{\mathbf{a}^{*}}-\hat{\mathbf{s}}^{\mathbf{S i}^{*}}\right\|^{2} \\
& =\underset{w_{k}}{\arg \min }\left\|\mathbf{x}^{\mathbf{a}^{*}}-\hat{\mathbf{w}}_{\mathbf{k}} \cdot \mathbf{i}\right\|^{2} \\
\hat{\mathbf{w}}_{\mathbf{k}}^{\mathbf{L S}} & =\left(\mathbf{i}^{H} \cdot \mathbf{i}\right)^{-1} \cdot \mathbf{i}^{H} \cdot \mathbf{x}^{\mathbf{a}^{*}}
\end{aligned}
$$

where the operator $[\cdot]^{H}$ represents the Hermitian transpose.

After the digital cancellation, the signal $\hat{x}(n)$ is going to be detected based on the channel state information (CSI). The transmitting antenna index and modulated QPSK symbols are then demodulated. The bit sequence information contained in them is concatenated to recover the input bitstream. 


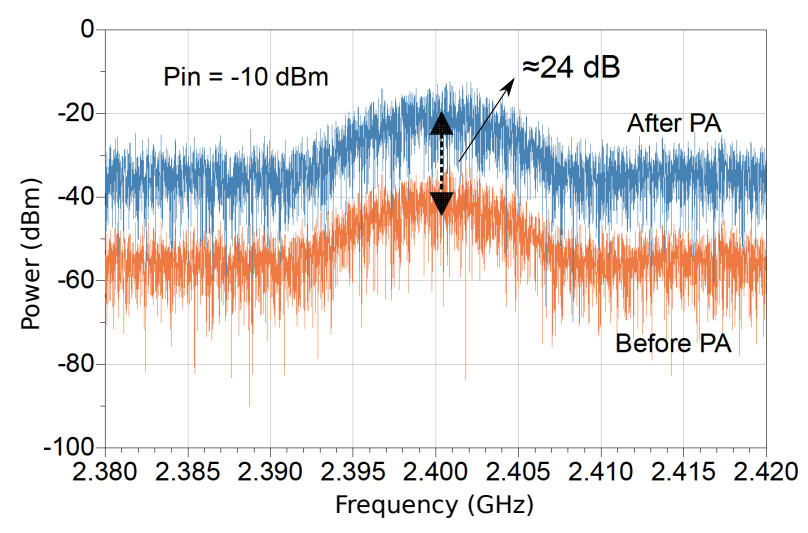

(a) $P_{\text {in }}=-10 \mathrm{dBm}$

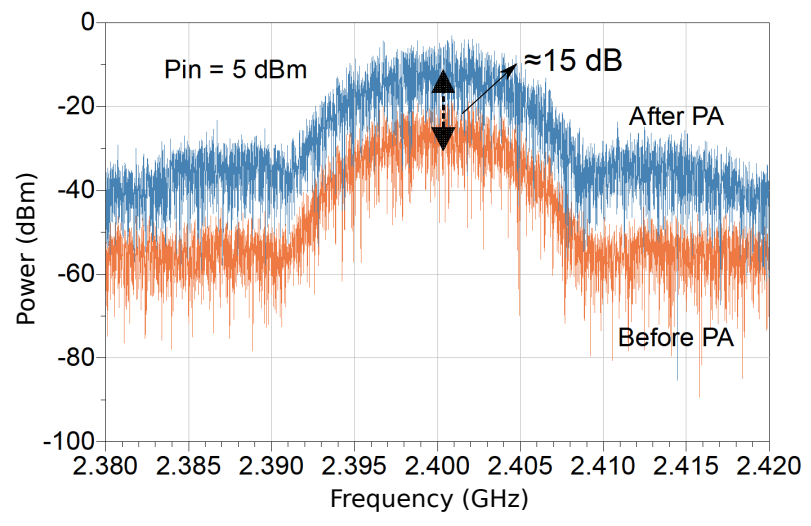

(b) $P_{\text {in }}=5 \mathrm{dBm}$

Fig. 3. Signal power before and after PA with $P_{i n}=-10 \mathrm{dBm}$ and $5 \mathrm{dBm}$

\section{System Performance Results}

In this section, a $2 \times 2$ FDSM system as presented in Fig. 1 is simulated based on an ADS-Matlab co-simulation framework. The system performance is evaluated in two steps: one is the BER performance with different INRs, and then we analyze its performance within the linear/nonlinear zone of the PA in terms of $\mathrm{Eb} / \mathrm{No}$ (energy per bit to noise power spectral density ratio). The main simulation parameters and the PA characteristics are shown in Table I. As seen in Fig. 2, the

TABLE I

SYSTEM PARAMETERS

\begin{tabular}{cc}
\hline \hline Parameter & Value \\
\hline Carrier frequency & $2.4 \mathrm{GHz}$ \\
Bandwidth & $20 \mathrm{MHz}$ \\
Symbol rate & $10 \mathrm{Mbit} / \mathrm{s}$ \\
Center output time step & $0.1 \mathrm{us}$ \\
Modulation type & $\mathrm{QPSK}$ \\
Crest factor & $7.6 \mathrm{~dB}$ \\
PA gain & $24 \mathrm{~dB}$ \\
$P_{\text {in }}$ & $-10 / 5 \mathrm{dBm}$ \\
$P_{\text {out }}$ & $14 / 20 \mathrm{dBm}$ \\
Impulse model & Root raised cosine \\
Filter type & Nyquist filter \\
Roll-off factor & 0.5 \\
\hline
\end{tabular}

simulated PA works at linear zone or nonlinear zone depending on the input signal power. Therefore, we apply $-10 \mathrm{dBm}$ and $5 \mathrm{dBm}$ as the input signal power in the linear zone and the nonlinear zone, respectively. The spectrum of the QPSK signals before and after PA in these two cases is shown in Fig. 3. We remark that the PA gain is about $24 \mathrm{~dB}$ with $P_{\text {in }}=-10$ $\mathrm{dBm}$ in the linear zone while PA gain is about $15 \mathrm{~dB}$ with $P_{i n}=5 \mathrm{dBm}$ in the nonlinear zone.

Firstly, three main scenarios are simulated to study the system BER performance with the variation of INR. One is the ideal case with total SIC and without nonlinear effects (perfect knowledge of the CSI). Another is the linear case and SI is estimated. The last is that the proposed digital canceller and the simulated PA are applied. In addition, SIC without nonlinearity cancellation and no SIC as two undesirable situations

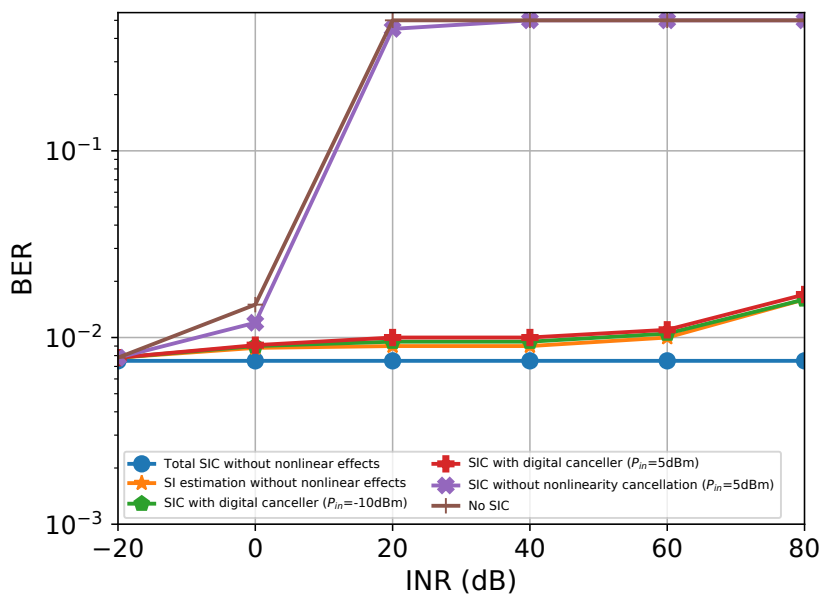

Fig. 4. BER performance under $\mathrm{Eb} / \mathrm{No}=15 \mathrm{~dB}$ (number of samples for $\mathrm{SI}$ estimation $\mathrm{N}=10000$ )

are simulated to compare the performance. The scenario of total SIC can be assumed to have the same performance of a single SM system. The degradation of BER in other scenarios is due to the incomplete elimination of SI.

The BER under $\mathrm{Eb} / \mathrm{No}=15 \mathrm{~dB}$ with different INRs is shown in Fig. 4. One can remark that with the proposed canceller, no matter which zone the PA is working in, the BER can be maintained at a level equivalent to the ideal one. Moreover, with the increase of INR, BER can be held at the original level and it will increase slightly when greater than 40 $\mathrm{dB}$. Besides, without taking the nonlinearities into account will cause a noticeable degradation, especially with a high INR. The number of samples $(N)$ for estimation can also be a main factor impacting BER. The BER performance in the linear zone $\left(P_{\text {in }}=-10 \mathrm{dBm}\right)$ and nonlinear zone $\left(P_{i n}=5 \mathrm{dBm}\right)$ with the variation of $\mathrm{Eb} / \mathrm{No}$ is given in Fig. 5. We can conclude that increasing $N$ will decline the BER to a certain extent but makes the estimator more complex. Even in the nonlinear 


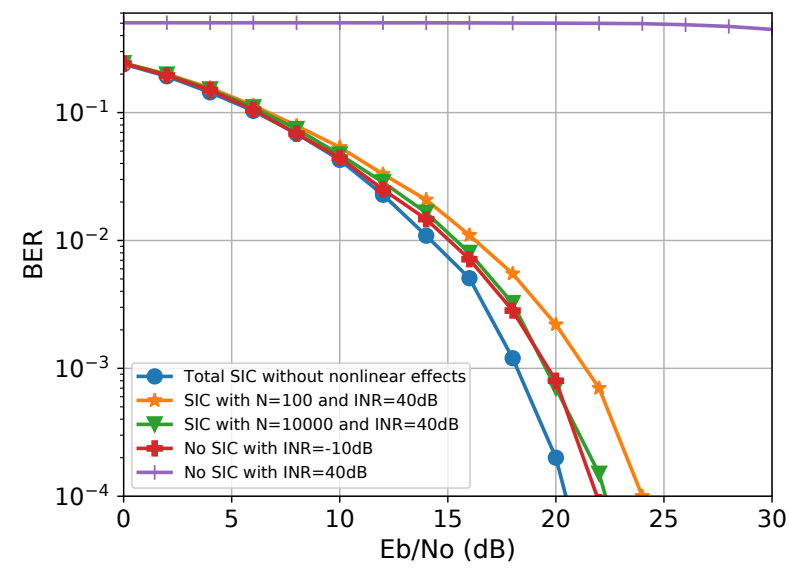

(a) $P_{\text {in }}=-10 \mathrm{dBm}$

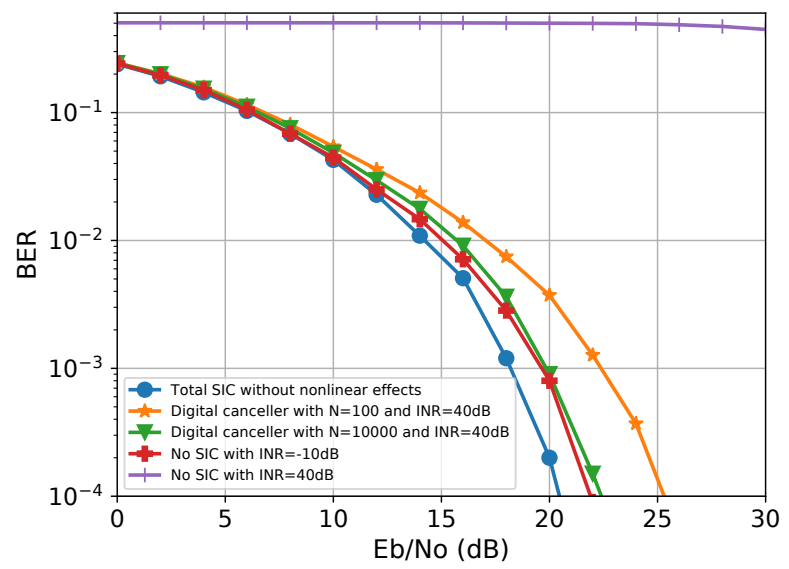

(b) $P_{\text {in }}=5 \mathrm{dBm}$

Fig. 5. BER performance under $P_{i n}=-10 \mathrm{dBm}$ and $5 \mathrm{dBm}$

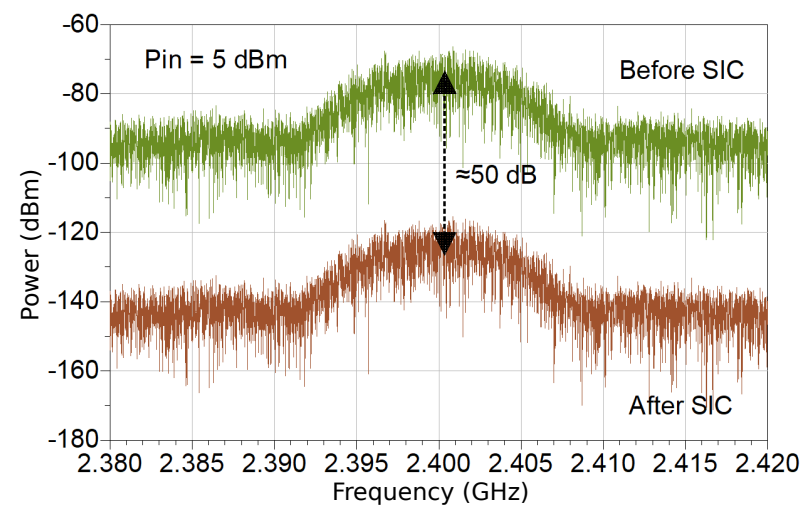

Fig. 6. SI power before and after digital canceller with INR $=40 \mathrm{~dB}$ and $P_{\text {in }}=5 \mathrm{dBm}$

zone, the proposed solution with $N=10000$ can cancel the strong SI (INR $=40 \mathrm{~dB})$ to the comparable level in the case of INR $=-10 \mathrm{~dB}$. That indicates SI is generally reduced to the approximate level as the thermal noise. This conclusion can also be drawn from Fig. 6, which indicates that the attenuation of SI power is about $50 \mathrm{~dB}$ with INR $=40 \mathrm{~dB}$ and $P_{\text {in }}=5$ $\mathrm{dBm}$.

\section{CONCLUSion}

In this paper, a FDSM system framework was developed, which includes the modules of antenna selection, modulation, PA, radio front-end of the receiver, digital canceller and detection. The nonlinear effects of a low-cost PA were modeled rigorously and a digital canceller was considered to suppress the SI signal taking into account PA nonlinearities. The reliability of this canceller was demonstrated by comparing the BER performance under different scenarios. The results show that with the considered canceller, when taking into account PA's nonlinear effects and at high SI level, the system performance will only slightly degrade and at a high-accuracy detection level. Also, the increase of the samples will consequently improve kind of the system performance. Finding a balance between the complexity of this canceller and high reliability is a problem worthy of in-depth research.

\section{REFERENCES}

[1] M. Duarte and A. Sabharwal, "Full-duplex wireless communications using off-the-shelf radios: Feasibility and first results," 44th Asilomar Conference on Signals, Systems and Computers, pp. 1558-1562, 2010.

[2] Z. Zhang, X. Chai, K. Long, A. V. Vasilakos, and L. Hanzo, "Full duplex techniques for 5G networks: Self-interference cancellation, protocol design, and relay selection," IEEE Communications Magazine, vol. 53, no. 5, pp. 128-137, 2015.

[3] N. Li, W. Zhu, and H. Han, "Digital interference cancellation in single channel, full duplex wireless communication," in 8th International Conference on Wireless Communications, Networking and Mobile Computing, 2012.

[4] M. Di Renzo, H. Haas, A. Ghrayeb, S. Sugiura, and L. Hanzo, "Spatial modulation for generalized MIMO: Challenges, opportunities, and implementation," Proceedings of the IEEE, vol. 102, no. 1, pp. 56-103, 2014.

[5] B. Jiao, M. Wen, M. Ma, and H. V. Poor, "Spatial modulated full duplex," IEEE Wireless Communications Letters, vol. 3, no. 6, pp. 641644, 2014.

[6] D. Korpi, T. Huusari, Y. Choi, L. Anttila, S. Talwar, and M. Valkama, "Digital self-interference cancellation under nonideal RF components: Advanced algorithms and measured performance," IEEE 16th International Workshop on Signal Processing Advances in Wireless Communications (SPAWC), pp. 286-290, 2015.

[7] D. Korpi, L. Anttila, and M. Valkama, "Nonlinear self-interference cancellation in MIMO full-duplex transceivers under crosstalk," EURASIP Journal on Wireless Communications and Networking, vol. 2017, no. 24, 2017.

[8] M. S. Sim, M. Chung, D. Kim, J. Chung, D. K. Kim, and C. Chae, "Nonlinear self-interference cancellation for full-duplex radios: From link-level and system-level performance perspectives," IEEE Communications Magazine, vol. 55, no. 9, pp. 158-167, 2017.

[9] Y. Zhou, F. Hutu, and G. Villemaud, "Impact of receiver non-idealities on a full duplex spatial modulation system performance," IEEE Wireless Communications Letters, vol. 9, no. 12, pp. 2083-2087, 2020.

[10] "CC2595 RF front-end transmit power amplifier for 2.4GHz ISM band systems," Texas Instruments Incorporated, Dallas, Texas, USA.

[11] D. Korpi, T. Riihonen, V. Syrjälä, L. Anttila, M. Valkama, and R. Wichman, "Full-duplex transceiver system calculations: Analysis of ADC and linearity challenges," IEEE Transactions on Wireless Communications, vol. 13, no. 7, pp. 3821-3836, 2014. 